\title{
Comment on: Scoping review and international multi-centre cohort study investigating teaching, knowledge and beliefs regarding physical activity as a health intervention among medical students: a comparison between Singapore and the UK
}

Singapore Med J 2019; 60(12): 655 https://doi.org/10.11622/smedj.2019161

Dear Sir,

As medical students in the United Kingdom (UK), we read with interest the article by Chew et al on physical activity (PA) as a health intervention. ${ }^{(1)}$ We were shocked to read that $74 \%$ of UK students had not received any formal teaching in discussing PA with patients and, even more so, that only $10 \%$ had observed a doctor giving PA advice. In contrast, we received formal training on this in our first year and are expected to put it into practice from the outset.

We follow the National Health Service England's Making Every Contact Count (MECC) initiative, ${ }^{(2)}$ which encourages staff to undertake brief health interventions during everyday consultations. The method used in MECC is the four 'As': ask, acknowledge, advise and assist. This simple and memorable structure helps clinicians to build confidence in broaching what can be an uncomfortable topic. Additionally, there is a pocketbook for health professionals that gives example statistics and facts to help standardise advice given to patients. $^{(3)}$

With such an effective tool, what then accounts for this variation in PA interventions in the UK? A major factor is low awareness of the MECC initiative, particularly among general practitioners (GPs), those best placed to support behavioural change. ${ }^{(4)}$ We feel that we can look to medical students for a practical solution: provided that students receive rigorous training in PA interventions, as we have, we may pass on our knowledge and awareness to staff in our GP placements. As the authors acknowledge, learning by observation is a powerful tool. Thus, the simple act of students practising PA interventions in clinics may be greatly influential.

Yours sincerely,

Laura Pattison ${ }^{1}$, James $\underline{\text { Mason }}^{1}$, Anvay Deshpande

${ }^{1}$ Warwick Medical School, Warwick University, Coventry, United Kingdom. Laura.pattison8@gmail.com

\section{References}

1. Chew EJC, Ho YN, Kee GJ, Sirisena D. Scoping review and international multi-centre cohort study investigating teaching, knowledge and beliefs regarding physical activity as a health intervention among medical students: a comparison between Singapore and the UK. Singapore Med J 2019; 60:642-51

2. Health Education England. Making Every Contact Count [online]. Available at: https://www.makingeverycontactcount.co.uk. Accessed September 5, 2019.

3. Health Education England. A Pocket Guide to Making Every Contact Count in Healthcare [online]. Available at: https://www.makingeverycontactcount.co.uk/ media/1040/012-mecc-pocketbook-for-healthcare-staff-june-15.pdf. Accessed September 5, 2019.

4. Keyworth C, Epton T, Goldthorpe J, Calam R, Armitage CJ. Are healthcare professionals delivering opportunistic behaviour change interventions? A multi-professional survey of engagement with public health policy. Implement Sci 2018; 13:122. 